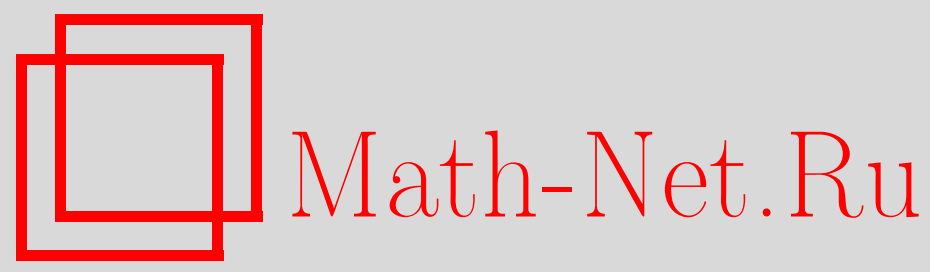

Д. С. Калюжный, О неравенстве фон Неймана для линейных матриц-функций нескольких переменных, Матем. заметки, 1998, том 64, выпуск 2, 218-223

DOI: https://doi.org/10.4213/mzm1389

Использование Общероссийского математического портала Math-Net.Ru подразумевает, что вы прочитали и согласны с пользовательским соглашением http://www . mathnet.ru/rus/agreement

Параметры загрузки:

IP : 54.224 .135 .184

26 апреля 2023 г., 05:43:42






\title{
О НЕРАВЕНСТВЕ ФОН НЕЙМАНА ДЛЯ ЛИНЕЙНЫХ МАТРИЦ-ФУНКЦИЙ НЕСКОЛЬКИХ ПЕРЕМЕННЫХ
}

\author{
Д. С. Калюжный
}

Доказана теорема о существовании трех коммутирующих сжатий в гильбертовом пространстве и линейной однородной матрицы-функции трех независимых переменных, для которых нарушается обобщенное неравенство фон Неймана.

Библиографория: 12 названий.

Дж. фон Нейман показал в [1], что для любого сжимающего линейного оператора $T$ в гильбертовом пространстве (т.е. $\|T\| \leqslant 1$ ) и любого многочлена $p(z)$ от одной переменной вьполнено неравенство

$$
\|p(T)\| \leqslant \max _{z \in \Delta}|p(z)|
$$

где $\Delta=\{z \in \mathbb{C}:|z| \leqslant 1\}$ - замкнутый единичный круг. Т. Андо [2] обобщил это неравенство на случай любых двух коммутирующих сжатий $T_{1}$ и $T_{2}$ в гильбертовом пространстве и любого многочлена $p\left(z_{1}, z_{2}\right)$ от двух независимых переменных:

$$
\left\|p\left(T_{1}, T_{2}\right)\right\| \leqslant \max _{z \in \Delta^{2}}|p(z)|
$$

(Здесь и далее, для любого натурального $N \Delta^{N}=\left\{z \in \mathbb{C}^{N}:\left|z_{k}\right| \leqslant 1, k=1, \ldots, N\right\}$ замкнутый единичный поликруг.) Однако, Н. Варопулос [3] показал, что уже для трех коммутирующих сжатий аналогичное неравенство, вообще говоря, не вьполняется, а именно: построил тройку коммутирующих сжимающих линейных операторов $T_{1}, T_{2}, T_{3}$ в некотором конечномерном гильбертовом пространстве и однородньй многочлен второй степени $p\left(z_{1}, z_{2}, z_{3}\right)$ от трех независимых переменных такие, что

$$
\left\|p\left(T_{1}, T_{2}, T_{3}\right)\right\|>\max _{z \in \Delta^{3}}|p(z)|
$$

Работа выполнена при поддержке фонда INTAS, грант № 93-0322ext. 
ЗАмечАниЕ 1. Степень многочлена в примере Варопулоса нельзя уменьшить. Действительно, пусть $T=\left\{T_{1}, \ldots, T_{N}\right\}$ - набор сжатий в некотором гильбертовом пространстве $\mathscr{H}$ со скалярным произведением $\langle\cdot, \cdot\rangle \mathscr{H}$ и пусть

$$
l\left(z_{1}, \ldots, z_{N}\right)=a_{0}+\sum_{k=1}^{N} a_{k} z_{k}
$$

- произвольный многочлен первой степени от $N$ независимых переменных, т.е. линейная скалярная функция в $\mathbb{C}^{N}$. Тогда

$$
\begin{aligned}
& \left\|l\left(T_{1}, \ldots, T_{N}\right)\right\|=\left\|a_{0} I_{\mathscr{H}}+\sum_{k=1}^{N} a_{k} T_{k}\right\|=\sup _{\|x\|=\|y\|=1}\left|\left\langle\left(a_{0} I_{\mathscr{H}}+\sum_{k=1}^{N} a_{k} T_{k}\right) x, y\right\rangle_{\mathscr{H}}\right| \\
& =\sup _{\|x\|=\|y\|=1} \mid a_{0}\langle x, y\rangle_{\mathscr{H}}+\sum_{k=1}^{N} a_{k}\left\langle T_{k} x, y \mathscr{H}\left|\leqslant \max _{\substack{\lambda \in \Delta \\
z \in \Delta^{N}}}\right| a_{0} \lambda+\sum_{k=1}^{N} a_{k} z_{k}\right| \\
& =\max _{|\zeta|=1}\left|a_{0} \zeta+\sum_{z \in \Delta^{N}}^{N} a_{k} z_{k}\right|=\max _{z \in \Delta^{N}}\left|a_{0}+\sum_{k=1}^{N} a_{k} z_{k}\right|=\max _{z \in \Delta^{N}}|l(z)|
\end{aligned}
$$

(мывоспользовались неравенством Коши-Буняковского-Шварца для оценки скалярного произведения и принципом максимума модуля для аналитических функций в круге). Отметим, что коммутативность набора $T$ здесь несущественна.

Однако, для матричнозначных многочленов (т.е. многочленов с матричными коэф(фициентами) от нескольких независимых переменньх можно рассмотреть понятие многочлена от нескольких коммутирующих сжатий [4]; тогда, как мы покажем, степень многочлена, для которого не выполняется соответствующее обобщенное неравенство фон Неймана, можно уменьшить до первой. Постановка этой задачи возникла при рассмотрении многопараметрических пассивных линейных систем рассеяния (определение таких систем см. в [5]), однако она имеет и самостоятельньй интерес.

Итак, пусть $T=\left\{T_{1}, \ldots, T_{N}\right\}$ - набор коммутируюших сжатий в гильбертовом пространстве $\mathscr{H}, P\left(z_{1}, \ldots, z_{N}\right)$ - матричнозначньй многочлен от $N$ независимых переменных, т.е.

$$
P(z)=\sum_{\substack{t \in \mathbb{Z}_{+}^{N} \\|t| \leqslant m}} A_{t} z^{t}, \quad z=\left(z_{1}, \ldots, z_{N}\right) \in \mathbb{C}^{N}
$$

где $\mathbb{Z}_{+}^{N}=\left\{t \in \mathbb{Z}^{N}: t_{k} \geqslant 0, k=1, \ldots, N\right\},|t|=\sum_{k=1}^{N} t_{k}$ для $t \in \mathbb{Z}_{+}^{N}, z^{t}=\prod_{k=1}^{N} z_{k}^{t_{k}}$, $A_{t} \in M_{n}(\mathbb{C})=\mathscr{L}\left(\mathbb{C}^{n}\right)$, т.е. мы будем отождествлять алгебру квадратных матриц порядка $n$ над полем $\mathbb{C}$ с $C^{*}$-алгеброй линейных операторов в конечномерном гильбертовом пространстве $\mathbb{C}^{n}$. Определим оператор

$$
P(T)=P\left(T_{1}, \ldots, T_{N}\right)=\sum_{\substack{t \in \mathbb{Z}_{+}^{N} \\|t| \leqslant m}} A_{t} \otimes T^{t} \in \mathscr{L}\left(\mathbb{C}^{n} \otimes \mathscr{H}\right) \cong \mathscr{L}\left(\mathscr{H}^{n}\right),
$$

здесь $T^{t}=\prod_{k=1}^{N} T_{k}^{t_{k}}$. Можно также рассматривать $P(T)$ как элемент $C^{*}$-алгебры $M_{n}(\mathbb{C}) \otimes \mathscr{L}(\mathscr{H}) \cong M_{n}(\mathscr{L}(\mathscr{H}))$ всех квадратных матрищ порядка $n$ над $\mathscr{L}(\mathscr{H})$, т.е. как матрицу с операторными элементами (см. [4]). Нам потребуется 
ОПРЕДЕЛЕНИЕ 1 [6]. Набор $U=\left\{U_{1}, \ldots, U_{N}\right\}$ коммутирующих унитарных операторов в гильбертовом пространстве $\mathscr{K}$ называется унитарной дилатачией набора $T=\left\{T_{1}, \ldots, T_{N}\right\}$ коммутирующих сжимающих операторов в гильбертовом пространстве $\mathscr{H} \subset \mathscr{K}$, если

$$
T^{t}=P_{\mathscr{H}} U^{t} \mid \mathscr{H}, \quad t \in \mathbb{Z}_{+}^{N},
$$

где $Р \mathscr{H}$ - ортогональный проектор в $\mathscr{K}$ на подпространство $\mathscr{H}$.

ЗАмЕчАниЕ 2. Из общей теоремы У. Арвесона о дилатациях (см. [7, с. 278]) следует, что набор $T=\left\{T_{1}, \ldots, T_{N}\right\}$ коммутирующих сжатий в гильбертовом пространстве обладает унитарной дилатацией тогда и только тогда, когда для любого матричнозначного многочлена $P(z)$ вида (1) вьполняется обобщенное неравенство фон Неймана

$$
\|P(T)\| \leqslant \max _{z \in \Delta^{N}}\|P(z)\|
$$

где $P(T)$ определяется равенством (2). В частности, для $N=1$ и $N=2$ это означает (см. [8] и [2]), что неравенство (3) всегда вьполняется.

Для $N=3$ сформулируем основное утверждение настоящей статьи.

ТЕОремА. В некотором конечномерном гильбертовом пространстве $\mathscr{H}$ существуют тройка $T=\left\{T_{1}, T_{2}, T_{3}\right\}$ коммутирующих сжсатий и тройка $A=$ $\left\{A_{1}, A_{2}, A_{3}\right\}$ квадратных матрии порядка $n, n>1$, над полем $\mathbb{C}$ такие, что линейная однородная матрииа-функиия

$$
L\left(z_{1}, z_{2}, z_{3}\right)=A_{1} z_{1}+A_{2} z_{2}+A_{3} z_{3}
$$

удовлетворяет неравенству

$$
\|L(T)\|>\max _{z \in \Delta^{3}}\|L(z)\|
$$

Для доказательства нам потребуются следующие определения.

ОПрЕДЕЛЕниЕ 2 (см., например, [9]). Пусть $\mathscr{A}$ и $\mathscr{B}-C^{*}$-алгебры. Линейное отображение $\varphi: \mathscr{A} \rightarrow \mathscr{B}$ называется положительным, если оно переводит положительные элементы $\mathscr{A}$ в положительные элементы $\mathscr{B}$, т.е. из $a \in \mathscr{A}, a \geqslant 0$ следует $\varphi(a) \geqslant 0$.

Пусть $\mathscr{S}$ - линейное подпространство в $C^{*}$-алгебре $\mathscr{A}$ (возможно, совпадающее с $\left.\mathscr{A}\right)$, $\mathscr{B}-C^{*}$-алгебра, $\varphi: \mathscr{S} \rightarrow \mathscr{B}$ - линейное отображение. Тогда для каждого натурального $n M_{n}(\mathbb{C}) \otimes \mathscr{A} \cong M_{n}(\mathscr{A})-C^{*}$-алгебра всех квадратных матриц порядка $n$ над $\mathscr{A}$, а $M_{n}(\mathbb{C}) \otimes \varphi-$ линейное подпространство этой $C^{*}$-алгебры. Если обозначить через $\mathrm{id}_{n}$ тождественное отображение $M_{n}(\mathbb{C})$ на себя, то $\varphi_{n}=\mathrm{id}_{n} \otimes \varphi$ есть линейное отображение $M_{n}(\mathbb{C}) \otimes \mathscr{S}$ в $M_{n}(\mathbb{C}) \otimes \mathscr{B}$, переводящее матрищы над $\mathscr{S}$ в матрицы над $\mathscr{B}$ применением линейного отображения $\varphi$ поэлементно.

ОПредЕЛЕНИЕ 3 [9]. Линейное отображение $\varphi: \mathscr{A} \rightarrow \mathscr{B}$ назьвается вполне положительным, если для всех натуральных $n$ отображения $\varphi_{n}$ положительны.

ОПРЕДЕЛЕНИЕ 4 [4]. Линейное отображение $\varphi: \mathscr{S} \rightarrow \mathscr{B}$ называется вполне сжимающ, им, если для всех натуральных $n$ отображения $\varphi_{n}$ сжимающие (т.е. $\left\|\varphi_{n}\right\| \leqslant 1$ ). 
ДокАЗАТЕЛЬСТво тЕоремЫ. Из замечания 2 следует, что искомая тройка сжатий $T$ не обладает унитарной дилатацией. Возьмем такую тройку сжатий из известного примера С. Парротта [10]. Пусть $\mathscr{X}$ - произвольное гильбертово пространство размерности больше 1 (т.е. $\mathscr{X}$ можно взять и конечномерным). Положим $\mathscr{H}=\mathscr{X} \oplus \mathscr{X}$. Зададим тройку $T=\left\{T_{1}, T_{2}, T_{3}\right\}$ сжимающих линейных операторов в гильбертовом пространстве $\mathscr{H}$ блочньми матрицами:

$$
T_{1}=\left(\begin{array}{cc}
0 & 0 \\
B_{1} & 0
\end{array}\right), \quad T_{2}=\left(\begin{array}{cc}
0 & 0 \\
B_{2} & 0
\end{array}\right), \quad T_{3}=\left(\begin{array}{cc}
0 & 0 \\
I_{\mathscr{X}} & 0
\end{array}\right),
$$

где $B_{1}$ и $B_{2}$ - произвольные некоммутирующие унитарные операторы в $\mathscr{X}, I \mathscr{X}-$ единичньй оператор в $\mathscr{X}$. Очевидно, $T_{k}$ - коммутирующие операторы, так как $T_{k} T_{j}=0$, $k, j=1,2,3$.

Пусть $\mathbb{T}^{N}=\left\{\lambda \in \mathbb{C}^{N}:\left|\lambda_{k}\right|=1, k=1, \ldots, N\right\}$ есть $N$-мерньй тор, $\mathscr{S}=\operatorname{span}\left\{\Lambda_{1}, \Lambda_{2}, \mathbf{I}\right\}$ - трехмерное линейное подпространство $C^{*}$-алгебры $C\left(\mathbb{T}^{2}\right)$ непрерывных комплекснозначных функций на двумерном торе $\mathbb{T}^{2}$, порожденное функциями

$$
\Lambda_{1}(\lambda)=\lambda_{1}, \quad \Lambda_{2}(\lambda)=\lambda_{2}, \quad \mathbf{I}(\lambda)=1
$$

Определим линейное отображение $\varphi: \mathscr{S} \rightarrow \mathscr{L}(\mathscr{X})$ следующим образом: для любых $\alpha, \beta, \gamma \in \mathbb{C}$ положим

$$
\varphi\left(\alpha \Lambda_{1}+\beta \Lambda_{2}+\gamma \mathbf{I}\right)=\alpha B_{1}+\beta B_{2}+\gamma I \mathscr{X}
$$

Покажем, что отображение $\varphi$ является сжимающим, но не является вполне сжимающим. Согласно замечанию 1

$$
\begin{aligned}
\left\|\varphi\left(\alpha \Lambda_{1}+\beta \Lambda_{2}+\gamma \mathbf{I}\right)\right\| & =\left\|\alpha B_{1}+\beta B_{2}+\gamma I \mathscr{X}\right\| \leqslant \max _{z \in \Delta^{2}}\left|\alpha z_{1}+\beta z_{2}+\gamma\right| \\
& =\max _{\lambda \in \mathbb{T}^{2}}\left|\alpha \lambda_{1}+\beta \lambda_{2}+\gamma\right|=\left\|\alpha \Lambda_{1}+\beta \Lambda_{2}+\gamma \mathbf{I}\right\|
\end{aligned}
$$

(мы воспользовались принципом максимума модуля для аналитических функций в бикруге). Таким образом, $\varphi$ - сжимающее отображение. Предположим теперь, что $\varphi$ является также вполне сжимающим отображением. Тогда по теореме 1.2.9 [4] оно продолжается до вполне положительного линейного отображения $\widetilde{\varphi}: C\left(\mathbb{T}^{2}\right) \rightarrow \mathscr{L}(\mathscr{X})$, причем $\widetilde{\varphi}(\mathbf{I})=I \mathscr{X} \cdot$ Такое отображение имеет вид [9]

$$
\widetilde{\varphi}(f)=P_{\mathscr{X}} \pi(f) \mid \mathscr{X}, \quad f \in C\left(\mathbb{T}^{2}\right),
$$

где $\pi$ - представление $C^{*}$-алгебры $C\left(\mathbb{T}^{2}\right)$ в некотором гильбертовом пространстве $\mathscr{Y} \supset \mathscr{X}$, а $P \mathscr{X}$ - ортогональньй проектор в $\mathscr{Y}$ на подпространство $\mathscr{X}$. Для любого $x \in \mathscr{X}$ имеем

$$
\|x\|=\left\|B_{k} x\right\|=\left\|\varphi\left(\Lambda_{k}\right) x\right\|=\left\|\widetilde{\varphi}\left(\Lambda_{k}\right) x\right\|=\left\|P_{\mathscr{X}} \pi\left(\Lambda_{k}\right) x\right\|, \quad k=1,2 .
$$

С другой стороны, $\pi\left(\Lambda_{k}\right)$ - унитарные операторы в пространстве $\mathscr{Y}$, так как $\Lambda_{k}-$ унитарные элементы $C^{*}$-алгебры $C\left(\mathbb{T}^{2}\right)$. Поэтому $\|x\|=\left\|\pi\left(\Lambda_{k}\right) x\right\|$. Таким образом,

$$
\left\|P_{\mathscr{X}} \pi\left(\Lambda_{k}\right) x\right\|=\left\|\pi\left(\Lambda_{k}\right) x\right\|,
$$


а это возможно только если $P_{\mathscr{X}} \pi\left(\Lambda_{k}\right) x=\pi\left(\Lambda_{k}\right) x$, т.е. $\pi\left(\Lambda_{k}\right) x \in \mathscr{X}$. Так как $x-$ произвольный элемент $\mathscr{X}$, отсюда получаем, что

$$
\pi\left(\Lambda_{k}\right)\left|\mathscr{X}=P_{\mathscr{X}} \pi\left(\Lambda_{k}\right)\right| \mathscr{X}=\widetilde{\varphi}\left(\Lambda_{k}\right)=\varphi\left(\Lambda_{k}\right)=B_{k}, \quad k=1,2
$$

Следовательно, имеем

$$
\begin{aligned}
B_{1} B_{2} & =\pi\left(\Lambda_{1}\right) \pi\left(\Lambda_{2}\right)\left|\mathscr{X}=\pi\left(\Lambda_{1} \Lambda_{2}\right)\right| \mathscr{X} \\
& =\pi\left(\Lambda_{2} \Lambda_{1}\right)\left|\mathscr{X}=\pi\left(\Lambda_{2}\right) \pi\left(\Lambda_{1}\right)\right| \mathscr{X}=B_{2} B_{1}
\end{aligned}
$$

что противоречит выбору операторов $B_{1}$ и $B_{2}$. Значит, $\varphi$ не является вполне сжимающим отображением, т.е. найдется натуральное $n>1$, для которого $\left\|\varphi_{n}\right\|>1$. Это, в свою очередь, означает, что найдется тройка $A=\left\{A_{1}, A_{2}, A_{3}\right\}$ квадратных матриц над $\mathbb{C}$ порядка $n$ таких, что

$$
\left\|\varphi_{n}\left(A_{1} \otimes \Lambda_{1}+A_{2} \otimes \Lambda_{2}+A_{3} \otimes \mathbf{I}\right)\right\|>\left\|A_{1} \otimes \Lambda_{1}+A_{2} \otimes \Lambda_{2}+A_{3} \otimes \mathbf{I}\right\|
$$

Исследуем отдельно левую и правую части этого неравенства. Имеем

$$
\begin{aligned}
& \left\|\varphi_{n}\left(A_{1} \otimes \Lambda_{1}+A_{2} \otimes \Lambda_{2}+A_{3} \otimes \mathbf{I}\right)\right\|=\left\|A_{1} \otimes B_{1}+A_{2} \otimes B_{2}+A_{3} \otimes I \mathscr{X}\right\| \\
& \quad=\left\|\left(I_{n} \otimes P_{\{0\} \oplus \mathscr{X}}\right)\left(A_{1} \otimes T_{1}+A_{2} \otimes T_{2}+A_{3} \otimes T_{3}\right) \mid \mathbb{C}^{n} \otimes(\mathscr{X} \oplus\{0\})\right\| \\
& \quad \leqslant\left\|A_{1} \otimes T_{1}+A_{2} \otimes T_{2}+A_{3} \otimes T_{3}\right\|,
\end{aligned}
$$

где $I_{n}$ - единичная матрица порядка $n, \mathscr{X} \oplus\{0\}$ и $\{0\} \oplus \mathscr{X}-$ подпространства в $\mathscr{H}=$ $\mathscr{X} \oplus \mathscr{X}$ всех таких векторов $h=x_{1} \oplus x_{2}$, у которых $x_{2}=0$ (соответственно $\left.x_{1}=0\right)$, а $P_{\{0\} \oplus \mathscr{X}}$ - ортопроектор в $\mathscr{H}$ на подпространство $\{0\} \oplus \mathscr{X}$. Правая часть неравенства (6)

$$
\left\|A_{1} \otimes \Lambda_{1}+A_{2} \otimes \Lambda_{2}+A_{3} \otimes \mathbf{I}\right\|=\max _{\lambda \in \mathbb{T}^{2}}\left\|A_{1} \lambda_{1}+A_{2} \lambda_{2}+A_{3}\right\|
$$

в силу изоморфизма $C^{*}$-алгебры $M_{n}(\mathbb{C}) \otimes C\left(\mathbb{T}^{2}\right)$ и $C^{*}$-алгебры $C\left(\mathbb{T}^{2}, M_{n}(\mathbb{C})\right)$ непрерывных на $\mathbb{T}^{2}$ матриц-функций размера $n \times n$ (см., например, [11, предложение 4.7.3]). Однако,

$$
\max _{\lambda \in \mathbb{T}^{2}}\left\|A_{1} \lambda_{1}+A_{2} \lambda_{2}+A_{3}\right\|=\max _{\zeta \in \mathbb{T}^{3}}\left\|A_{1} \zeta_{1}+A_{2} \zeta_{2}+A_{3} \zeta_{3}\right\|=\max _{z \in \Delta^{3}}\left\|A_{1} z_{1}+A_{2} z_{2}+A_{3} z_{3}\right\|
$$

согласно принципу максимума для аналитических матриц-функций нескольких переменных (см., например, [12]).

Таким образом, окончательно получаем неравенство

$$
\left\|A_{1} \otimes T_{1}+A_{2} \otimes T_{2}+A_{3} \otimes T_{3}\right\|>\max _{z \in \Delta^{3}}\left\|A_{1} z_{1}+A_{2} z_{2}+A_{3} z_{3}\right\|
$$

которое совпадает с неравенством (5) для линейной однородной матрицы-функции (4). Теорема доказана. 


\section{СПИСОК ЦИТИРОВАННОЙ ЛИТЕРАТУРЫ}

[1] Von Neumann J. Eine Spectraltheorie für allgemeine Operatoren eines unitären Raumes // Math. Nachr. 1951. V. 4. P. 258-281.

[2] Ando T. On a pair of commutative contractions // Ann. of Math. (2). 1963. V. 24. P. 88-90.

[3] Varopoulos N. Th. On an inequality of von Neumann and an application of the metric theory of tensor products to operator theory // J. Funct. Anal. 1974. V. 16. P. 83-100.

[4] Arveson W. B. Subalgebras of $C^{*}$-algebras // Acta Math. 1969. V. 123. P. 141-224.

[5] Kalyuzhniy D. Multiparameter passive scattering linear stationary dynamic systems (discrete case) // Mark Krein International Conference "Operator theory and applications" (Odessa, August 18-22, 1997). Abstracts. Odessa, 1997.

[6] Сёкевальфи-Надь Б., Фояш Ч. Гармонический анализ операторов в гильбертовом пространстве. М.: Мир, 1970.

[7] Arveson W. B. Subalgebras of $C^{*}$-algebras. II // Acta Math. 1972. V. 128. P. 271-308.

[8] Szökefalvi-Nagy B. Sur les contractions de l'espace de Hilbert // Acta Sci. Math. (Szeged). 1953. V. 15. P. 87-92.

[9] Stinespring W. Positive functions on $C^{*}$-algebras // Proc. Amer. Math. Soc. 1955. V. 6. P. 211-216.

[10] Parrott S. Unitary dilations for commuting contractions // Pacific J. Math. 1970. V. 34. P. 481-490.

[11] Takesaki M. Theory of Operator Algebras. V. 1. New York-Heidelberg-Berlin: Springer, 1979.

[12] Шварц Л. Анализ. Т. 2. М.: Мир, 1972.

Одесская государственная академия строительства и архитектуры

Поступило

E-mail: Dmitriy.Kalyuzhniy@p25.f61.n467.z2.fidonet.org

16.10 .97 